\title{
An Exploratory Analysis of the Geographical Distribution of Trauma Incidents in Shenzhen, China
}

(Original Scientific Reports; word count - 2289)

Authors: Gui Xi ZHANG, MBBS, MHSM, MTS, Department of Surgery, the University of Hong Kong-Shenzhen Hospital, Shenzhen, China Joe King Man FAN, MBBS, FRCSEd, FCSHK, FHKAM, Department of Surgery, the University of Hong Kong-Shenzhen Hospital, Shenzhen, China; Department of Surgery, Li Ka Shing Faculty of Medicine, the University of Hong Kong, Hong Kong Special Administrative Region, China

Fion Siu Yin CHAN, MBBS, FRCSEd, FCSHK, FHKAM, Department of Surgery, the University of Hong Kong-Shenzhen Hospital, Shenzhen, China; Department of Surgery, Li Ka Shing Faculty of Medicine, the University of Hong Kong, Hong Kong Special Administrative Region, China

Gilberto Ka Kit LEUNG, MBBS, BSc, PhD, MS, FRCSEd, FCSHK, FHKAM, Department of Surgery, the University of Hong Kong-Shenzhen Hospital, Shenzhen, China; Department of Surgery, Li Ka Shing Faculty of Medicine, the University of Hong Kong, Hong Kong Special Administrative Region, China

Chung Mao LO, MBBS, MS, FRCS, FRACS, FACS, FHKAM, FCSHK, Department of Surgery, the University of Hong Kong-Shenzhen Hospital, Shenzhen, China; Department of Surgery, Li Ka Shing Faculty of 
Medicine, the University of Hong Kong, Hong Kong Special Administrative Region, China

Yi Min YU, MD, Shenzhen “120” Emergency Medical Services Center, Shenzhen, China

Hong ZHANG, MD, Shenzhen “120” Emergency Medical Services Center, Shenzhen, China

Susan I. BRUNDAGE, MD, MPH, FACS, Centre for Trauma Sciences, Queen Mary University of London, London; s.brundage@qmul.ac.uk Jan Olaf JANSEN, FRCS FFICM, Departments of Surgery and Intensive Care Medicine, Aberdeen Royal Infirmary \& Health Services Research Unit, University of Aberdeen; jan.jansen@abdn.ac.uk 


\begin{abstract}
Background

The city of Shenzhen, China, is planning to establish a trauma system. At present, there are few data on the geographical distribution of incidents, which is key to deciding on the location of trauma centers. The aim of this study was to perform a geograpical analysis in order to inform the development of a trauma system in Shenzhen.
\end{abstract}

\title{
Methods
}

Retrospective analysis of trauma incidents attended by Shenzhen Emergency Medical Services (EMS) in 2014. Data were obtained from Shenzhen EMS. Incident distribution was explored using dot- and Kernel density estimate maps. Clustering was determined using the nearest neighbour index. The type of healthcare facilities which patients were taken to was compared against patients' needs, as assessed using the Field Triage Decision Scheme.

\section{Results}

There were 49082 recorded incidents. 3513 were classed as major trauma. Mapping demonstrates that incidents predominantly occurred in the western part of Shenzhen, with identifiable clusters. Nearest neighbour index was 0.048 . Of patients deemed to have suffered major trauma, $8.5 \%$ were taken to a teaching hospital, $13.6 \%$ to a regional hospital, $42.6 \%$ to a community hospital and $35.3 \%$ to a private hospital. The proportions of Step 1 or 2 negative patients were almost identical.

\section{Conclusion}

The majority of trauma patients, including trauma patients who are at greater likelihood of severe injury are taken to regional and community hospitals. There are areas with identifiable concentrations of volume, which should be considered for the siting of highlevel trauma centers, although further modeling is required to make firm 
recommendations.

Key words: Geographical Analysis; Trauma Incidents; Trauma System 


\section{INTRODUCTION}

Trauma represents a significant and growing public health concern in China. ${ }^{1} \mathrm{An}$ analysis by the World Health Organisation has found that, in urban areas, mortality from injury has increased over the past five decades ${ }^{2}$, and a recent systematic review has identified transport-related accidents, falls, and industrial injuries as the most common mechanisms of injury ${ }^{3}$. Road traffic injuries are particularly problematic. ${ }^{4}$

Trauma systems - clinical networks for the treatment of trauma patients, encompassing prehospital care, acute care and reconstruction in specialist centres, and rehabilitation ${ }^{5}$ - have been shown to reduce both mortality and disability ${ }^{6-7}$, but trauma system development in China remains in its infancy. ${ }^{8-9}$

Shenzhen is a major city in Guangdong province, in southeast China, located immediately north of the Hong Kong Special Administrative Region. In 1979, it was designated as China's first Special Economic Zone, and became one of the fastestgrowing cities in the world. Today, Shenzhen has a population of 10.8 million residents, with a further 8 million residents in the surrounding metro area. ${ }^{10}$

Trauma is increasingly recognised as a major public health issue in Shenzhen, and policymakers, as well as the Chinese Medical Association Trauma Committee, are considering the development of a citywide trauma system. Such a system requires the designation of specialist trauma centres, which require sufficient case volumes to develop the institutional expertise, and justify the staffing and facilities required, to improve outcomes. However, trauma centres must also be accessible, so that time to definitive care is minimised. ${ }^{5}$ Geospatially effective trauma systems ensure that the majority of patients can reach an appropriate level care in the shortest possible time.

At present, there is little information on the geographical distribution of trauma incidents in Shenzhen, and which hospitals patients with suspected serious injuries are taken to. The aim of this study was to characterize the spatial distribution of trauma incidents in Shenzhen, and to analyze the level of destination healthcare facilities which patients 
are taken to, with a view to informing the development of a trauma system. 


\section{METHODS}

\section{Study design}

This is a retrospective study of trauma incidents attended by "120" Emergency Medical Services (EMS) in Shenzhen, between 1 January and 31 December 2014. The study was approved by Shenzhen EMS and Queen Mary University of London (reference QMREC1586a).

\section{Setting}

The city of Shenzhen covers an area of $1996 \mathrm{~km}^{2}$, extending for approximately $80 \mathrm{~km}$ from West to East, and approximately $40 \mathrm{~km}$ from North to South, at its widest points. It is comprised of six administrative districts (Luohu, Futian, Nanshan, Baoan, Longgang, and Yantian) and four new districts (Guangming, Pingshan, Longhua, and Dapeng). ${ }^{1}$ Shenzhen's health system has 76 hospitals, of which 5 are university hospitals (with approximately $1500-2000$ beds each), one of which is the children's hospital, 9 are regional hospitals (approximately $800-1000$ beds each), 25 are community hospitals (approximately 400-500 beds each), 31 are private hospitals (with usually less than 200 beds), and 6 are specialist hospitals (which do not receive trauma patients) (figure 1). The five university hospitals are all located in close proximity, in the Futian and Luohu area.

Prehospital care is the responsibility of the Shenzhen "120" EMS network, which receives all incoming calls, and is responsible for the dispatch of ambulances. At present, there is no prehospital trauma triage, and patients are taken to the nearest facility, regardless of the nature of their injuries (There is some kind of temporary triage when multiple casualty happens or a disaster happens). Although the ambulances are owned by Shenzhen "120" EMS, they are staffed by doctors and nurses who are nearly employed by hospitals, who in general prefer to take patients to "their" hospital, adding further complexity to tasking. 


\section{Data source and geocoding}

Data were obtained from Shenzhen "120" EMS, who maintains electronic records of all incidents attended. We extracted anonymised demographic and physiological data, as well as diagnoses and other clinical details, and incident locations. Data were collated using Microsoft Excel for Mac 2011, Version 14.4.6 (Microsoft, Redmond, WA). The EMS registry records both diagnoses and incident locations as free text. The identification of incidents involving trauma was facilitated by the fact that virtually all injury-related diagnoses contain the word "伤” (injury). In order to further evaluate the geographical distribution of incidents, the incident location free text fields were geocoded into longitude and latitude using a combination of automated (Xgeocoding, http://www.gpsspg.com/xGeocoding/) and manual geocoding, using google maps.

\section{Classification of incidents}

As there is no pre-hospital triage system in place, we attempted to identify the most severely injured patients, by retrospectively applying the criteria of step 1 and step 2 of the American College of Surgeons/Center for Disease Control's Field Triage Decision Scheme. ${ }^{11}$ Step 1 relates to the patient's physiology, and recommends that those with a Glasgow Coma Scale of $<14$, a systolic blood pressure of $<90 \mathrm{mmHg}$, a respiratory rate of $<10$ or $>29$ should be taken to the highest level of trauma center. Step 2 lists key injuries, including penetrating torso and junctional trauma; flail chest; two or more proximal long bone fractures; crushed, degloved, or mangled extremities; amputation proximal to wrist or ankle; pelvic fractures; open or depressed skull fractures; and paralysis which should similarly be managed in the highest level of facility available.

\section{Spatial analysis}

The spatial analysis was conducted using QGIS 2.12.1, an open-source geographic information system (http://www.qgis.org/en/site/). Incident density distribution was explored graphically, using Kernel density estimate ("heat”) maps. Global clustering was quantified using the Nearest Neighbour Index, which compares the average 
distance between each incident and its nearest neighbor, and the average distance to what would have been expected in a random nearest neighbor distribution. An index of 1 indicates that there is no discrepancy between the expected distances in a random distribution and the measured distances in the actual distribution. An index of less than 1 indicates that the incidents are more clustered than would be expected from a random distribution.

\section{Classification of receiving hospitals}

Hospital which patients were taken to were classified as university hospitals, regional hospitals, community hospitals, private hospitals, and specialist hospitals. 


\section{RESULTS}

\section{Baseline characteristics}

Over the 12-month duration of the study, there were 118850 emergency calls, of which $49082(41.3 \%)$ related to injury. The baseline characteristics of the study group as a whole, as well as the subgroups, are shown in table 1 . The majority of patients $(71.6 \%)$ were male, and the median age was 32 years (interquartile range $25-44$ years). Of these, $3513(7.1 \%)$ patients were retrospectively classified as having met the criteria for step 1 or 2 of the FTDS, and thus having a high likelihood of having suffered major trauma. A slightly higher proportion of these $(75.2 \%)$ were male, compared with patients who did not meet the criteria for step 1 or 2 of the FTDS $\left(71.4 \%, \mathrm{p}<0.01, \mathrm{Chi}^{2}\right.$ test). The principal causes of injury were traffic injuries, assaults and falls.

\section{Destination healthcare facility}

Table 2 shows the level of the receiving medical facility. The majority of patients were taken to community $(20297,41.4 \%)$ or private hospitals (18 $013,36.7 \%)$. Only 3877 patients $(7.9 \%)$ were taken directly to university hospitals, or regional hospitals $(6895$, 14.0\%). Furthermore, the proportion of patients who went to university, regional, community, or private hospitals did not vary with the retrospectively determined severity of the injury ( $p=0.124, \mathrm{Chi}^{2}$ test, 3 degrees of freedom). Of the 3513 patients who met the criteria for step 1 and 2 of the FTDS, 299 (8.5\%) were taken to a university hospital, $479(13.6 \%)$ to a regional hospital, and $1496(42.6 \%)$ to a community hospital.

\section{Spatial analysis}

Figure 2 shows a dot map of the geographical distribution of the 3513 incidents classified as major trauma. There are concentrations of incidents particularly in the western and central parts of Shenzhen, along the border with Hong Kong. The nearest neighbour index was 0.048 , indicating a high degree of clustering. Kernel density estimation (figure 3) confirms these findings. Incident density is lower in the eastern 
parts of Shenzhen, particularly in Dapeng and Pingshan districts. In the western and central parts of the city, there are areas of high incident density in Luohu, Futian, Nanshan, Longhua, Longgang and Baoan districts. Figures 3 also shows the location of university hospitals, regional hospitals, and community hospitals in Shenzhen, and demonstrates that the volume concentrations in the northern parts of Baoan and in Guangming are only associated with community hospitals. 


\section{DISCUSSION}

The study has explored the geographical distribution of trauma incidents in Shenzhen, a major city in China, and the level of destination healthcare facility which trauma patients in the city are currently taken to.

\section{The need for a trauma system}

The results demonstrate that the majority of trauma patients, including trauma patients who are at greater likelihood of severe injury - as assessed using the Field Triage Decision Scheme - are taken to regional and community hospitals. This is an important finding. These hospitals are less likely to have the facilities, or personnel, to treat serious injuries, although it is important to recognise that being a university hospital does not equate to being a trauma centre.

\section{The need for prehospital trauma triage}

The results furthermore suggest that the current policy of taking patients to the nearest hospital is indeed being followed, and that there is no "surreptitious triage" taking place, whereby prehospital personnel might be taking patients whom they perceive to be more severely injured to different hospitals. These findings mirror those from other settings without a trauma system, such as Scotland, ${ }^{12-13}$ and indicate the need for the development of city-wide prehospital trauma triage. There are many such system, of which the Field Triage Decision Scheme is the most widely used. It would seem relatively straightforward to adopt the protocol, although implementation will require extensive education and training, to ensure that it is applied correctly. Similarly, the quality of prehospital trauma should subsequently be continuously assessed, by linking triage data, from the EMS network, and trauma registry data, from the trauma centres.

\section{Trauma centre designation}

The analysis of spatial patterns of injury can help to inform the development of trauma networks ${ }^{14-15}$, and this analysis demonstrates that, as in many other settings, ${ }^{16}$ the geographical distribution of incidents involving trauma is not even, and that there are 
identifiable concentrations of volume. Incident density is greater in the western part of Shenzhen, in Baoan, Nanshan, Futian, Longhua, Guangming, and the western part of Longgang, than in eastern districts of Dapeng, Yantian, Pingshan, and eastern Longgang. This distribution does, to some extent, reflect the population distribution. Although it is possible and straightforward to correct for population size, it is important to recognise that the design of a trauma system must reflect case volume, rather than incidence. It is possible to have areas of high incidence, which nevertheless contribute few patients.

Our study shows that trauma incidents in Shenzhen are highly clustered, which may facilitate the designation of hospitals as trauma centres, if suitable hospitals are located near the areas which contribute large numbers of patients. It is important to recognise that the Shenzhen metro area is relatively small, but densely populated. Its trauma system will thus be predominantly "urban", characterised by short distances, but also heavy traffic. In the more populated western parts of the city, where most of the trauma incidents were recorded, drive time, even with traffic, to the centre is usually less than an hour, often regarded as the maximum access time threshold for a trauma system. (Although there is little evidence for this threshold, albeit widely used.) It may therefore be possible to concentrate major trauma services in relatively few hospitals, thus ensuring sufficient volume to develop institutional expertise. Access times from the eastern parts of the city, and the Dapeng peninsula in particular, are considerably longer, although this study did not consider access times, and these will require further study.

At present, there are nine regional medical centers in Shenzhen city, although this number is set to increase to 17 by $2020 .{ }^{17}$ Not all of these hospitals can, or should be, designated as level I centers. The mapping of trauma volumes conducted as part of this study, together with the locations of existing hospitals, is a useful exploratory tool for initiating discussions regarding the designation of trauma centres. However, such decisions cannot be based on visual impressions alone, and further analysis - such as 
used by the GEOS study ${ }^{18}$ from Scotland, or the TRAMAH model ${ }^{19}$ - is required before firm recommendation can be made. Furthermore, the designation process should not only consider level I centers, but also level II and III centers, in keeping with the philosophy of an inclusive trauma network. Given the relatively small size of Shenzhen (about $80 \mathrm{~km}$ wide from west to east and $40 \mathrm{~km}$ from north to south), it should be possible to limit the number of high-level centres.

\section{Limitation and strengths}

This study has limitations, the most important being its retrospective design. Although the distinction into patients who would, or would not, have met the criteria for step 1 and step 2 of the Field Triage Decision Scheme is helpful, it is somewhat artificial. Patient flow should be based on triage, and triage represents a clinical decision, based on information available at the time, and provider judgement. The study's principal strength is its inclusive, population-based design. 


\section{CONCLUSION}

This analysis adds to our understanding of trauma care in Shenzhen. While it cannot recommend a specific configuration of trauma system, it has demonstrated that there is a need for such a network, and that the spatial distribution of incidents should facilitate the eventual designation of trauma centres, as part of an inclusive trauma system. 


\section{DISCLOSURES}

The Health Services Research Unit receives funding from the Chief Scientist Office of the Scottish Government Health and Social Care Directorates. The opinions expressed in this article are those of the authors alone. 


\section{REFERENCES}

[1] China health statistical yearbook. (http://www.nhfpc.gov.cn/zwgkzt/tinj/list.shtml, access on 04/06/2016)

[2] Zhao Jinbao, Deng Wei. Traffic accidents on expressways: new treat to China. Traffic injury prevention, 2012, Volume 13, Issue 3, Page 230.

[3] Michael Fitzharris, James Yu, Naomi Hammond, et al. Injury in China: a systematic review of injury surveillance studies conducted in Chinese hospital emergency departments. BMC emergency medicine, 2011, Volume 11, Issue 7, Page 18.

[4] Ma Sai, Li Qingfeng, Zhou Maigeng, et al. Road traffic injury in China: a review of national data sources. Traffic injury prevention, 2012, Volume 13 Suppl 1, Page 57.

[5] Latifi Rifat, Ziemba Michelle, Leppäniemi A, et al. Trauma system evaluation in developing countries: Applicability of American College of Surgeons/Committee on Trauma (ACS/COT) Basic Criteria. World Journal of Surgery, Aug. 2014, Volume 38, Issue 8, Pages 1898-1904.

[6] Gabbe Belinda J, Simpson Pam M, Sutherland AM, et al. Improved functional outcomes for major trauma patients in a regionalized, inclusive trauma system. Annals of surgery, April 2012. Volume 255, Issue 6, Pages: 1009-1015.

[7] Mackenzie Ellen J, Rivara Frederick P, Jurkovich Gregory J, et al. A National Evaluation of the Effect of Trauma-Center Care on Mortality. The New England Journal of Medicine, Jan. 2006. Volume 354, Issue 4, Pages 366-378.

[8] Dai Kerong, Xu Zhaochun, Zhu Liulong, et al. Trauma care systems in China. Injury, 2003, Volume 34, Issue 9, Pages 664-668.

[9] Zong Zhaowen, Li Nan, Cheng Tianmin, et al. Current state and future 
perspectives of trauma care system in Mainland China. Injury, 2011. Volume 42, Issue 9, Pages 874-878.

[10] Shenzhen [Internet]. Shenzhen. [cited 2016, Aug 7]. Retrieved from: https://en.wikipedia.org/wiki/Shenzhen

[11] Sasser Scott M, Hunt Richard C, Faul Mark, et al. Guidelines for field triage of injured patients: recommendations of the National Expert Panel on Field Triage, 2011. MMWR. Recommendations and reports. 2012. Volume 61, Issue rr-1, Page 1.

[12] Sudlow Alexis, McConnell Nicola, Egan Gerry, et al. Destination healthcare facility of patients with suspected traumatic brain injury in Scotland: Analysis of prehospital data. Injury, 2013. Volume 44, Issue 9, Pages 1237-1240.

[13] Peach Christopher M, Morrison Jonathan J, Apodaca Amy N, et al. Destination healthcare facility of shocked trauma patients in Scotland: analysis of transfusion and surgical capability of receiving hospitals. The SURGEON: Journal of the Royal College of Surgeons of Edinburgh and Ireland, 2013. Volume 11, Issue 5, Pages: 272-277.

[14] Jansen Jan O, Morrison Jonathan J, Wang Handing, et al. Feasibility and utility of population-level geospatial injury profiling: prospective, national cohort study. Journal of Trauma and Acute Care Surgery, May 2015. Volume 78, Issue 5, Pages: 962-969.

[15] Schuurman, Nadine, Hameed S Morad, et al. The spatial epidemiology of trauma: the potential of geographic information science to organize data and reveal patterns of injury and services. Canadian Journal of Surgery. Volume 51, Issue 5, Page: 389.

[16] Jansen JO, Morrison JJ, Wang H, et al. Feasibility and utility of population-level geospatial injury profiling: prospective, national cohort study. J Trauma Acute Care Surg. United States; 2015May;78(5):962-9. 
[17] http://www.tgtg365.com/article.php?id=1730 (access on 28/05/2016)

[18] Jansen, JO, Morrison JJ, Wang H, et al. Optimizing trauma system design: the GEOS (Geospatial Evaluation of Systems of Trauma Care) approach. J Trauma Acute Care Surg. 2014 Apr;76(4):1035-40.

[19] Branas CC, Mackenzie EJ, Williams JC, et al. Access to trauma centers in the United States. JAMA. 2005 Jun 1;293(21):2626-33. 


\section{TABLES}

Table 1. Characteristics of study population

\begin{tabular}{|l|c|c|c|}
\hline & All patients & $\begin{array}{c}\text { FTDS Step 1/2 } \\
\text { positive patients }\end{array}$ & $\begin{array}{c}\text { FTDS Step 1/2 } \\
\text { negative patients }\end{array}$ \\
\hline $\mathrm{n}$ & 49082 & 3513 & 45569 \\
\hline Age, years, median (IQR) & $32(25-44)$ & $32(24-44)$ & $32(25-44)$ \\
\hline Male gender, $\mathrm{n}(\%)$ & $35141(71.6 \%)$ & $2644(75.2 \%)$ & $32554(71.4 \%)$ \\
\hline Mechanism & & & \\
\hline Traffic collision, $\mathrm{n}(\%)$ & $19550(39.8 \%)$ & $1553(44.2 \%)$ & $17997(39.5 \%)$ \\
\hline Assaults, $\mathrm{n}(\%)$ & $14816(30.2 \%)$ & $956(27.2 \%)$ & $13860(30.4 \%)$ \\
\hline Falls, $\mathrm{n}(\%)$ & $6847(14.0 \%)$ & $613(17.4 \%)$ & $6234(13.7 \%)$ \\
\hline Other causes, $\mathrm{n}(\%)$ & $7869(16.0 \%)$ & $391(11.1 \%)$ & $7478(16.4 \%)$ \\
\hline
\end{tabular}


Table 2. Level of receiving medical facility

\begin{tabular}{|l|c|c|c|}
\hline & All patients & $\begin{array}{c}\text { FTDS Step 1/2 } \\
\text { positive patients }\end{array}$ & $\begin{array}{c}\text { FTDS Step 1/2 } \\
\text { negative patients }\end{array}$ \\
\hline University hospital $^{*}$ & $3877(7.9 \%)$ & $299(8.5 \%)$ & $3578(7.9 \%)$ \\
\hline Regional hospital & $6895(14.0 \%)$ & $479(13.6 \%)$ & $6416(14.1 \%)$ \\
\hline Community hospital & $20297(41.4 \%)$ & $1496(42.6 \%)$ & $18801(41.3 \%)$ \\
\hline Private hospital & $18013(36.7 \%)$ & $1239(35.3 \%)$ & $16774(36.8 \%)$ \\
\hline
\end{tabular}

* Includes children's hospital 
FIGURES 
Figure 1. Map of Shenzhen area (shaded pink)

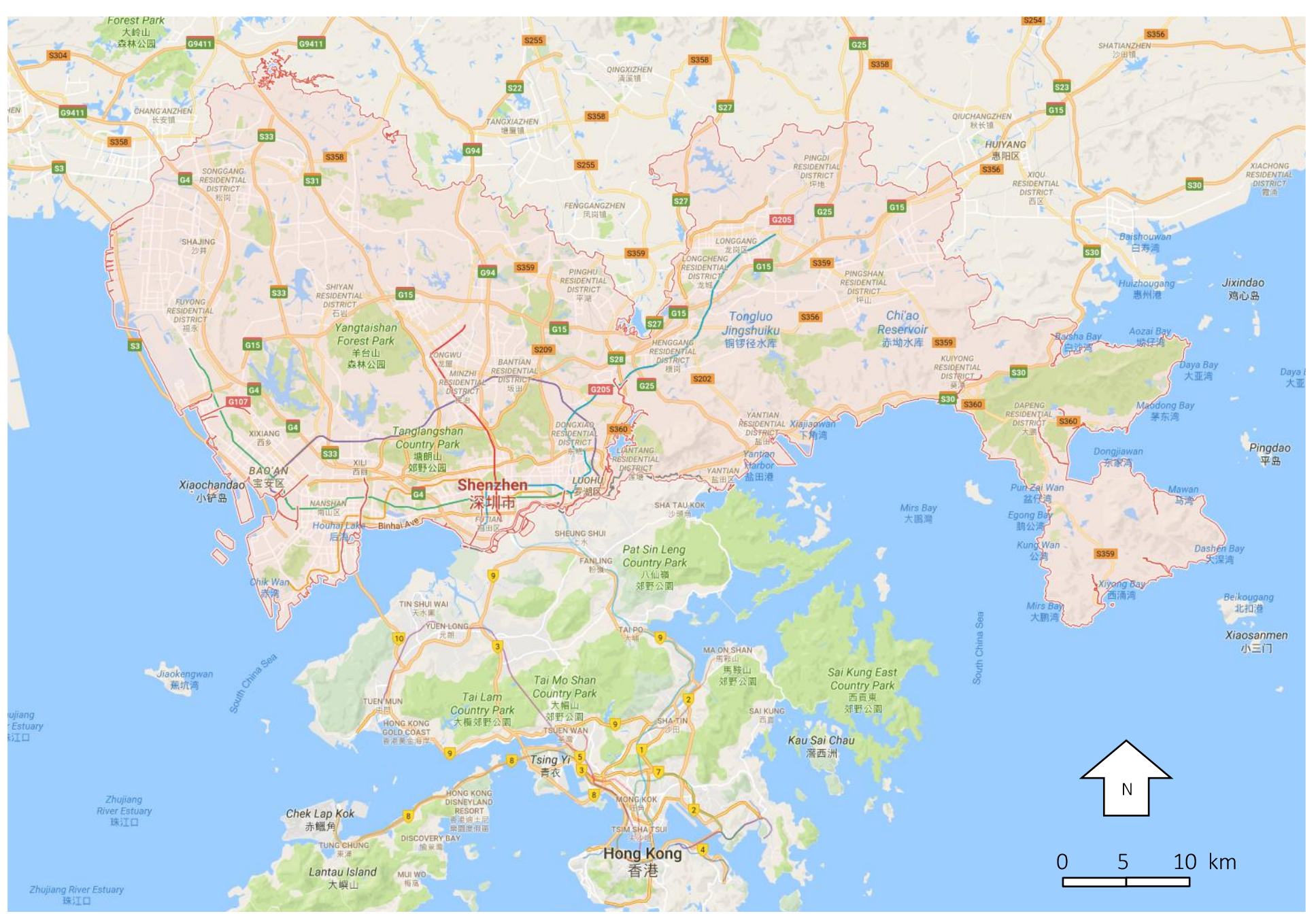


Figure 2. Dot map of incidents which, in retrospect, met criteria for Step 1 or 2 of the Field Triage Decision Scheme

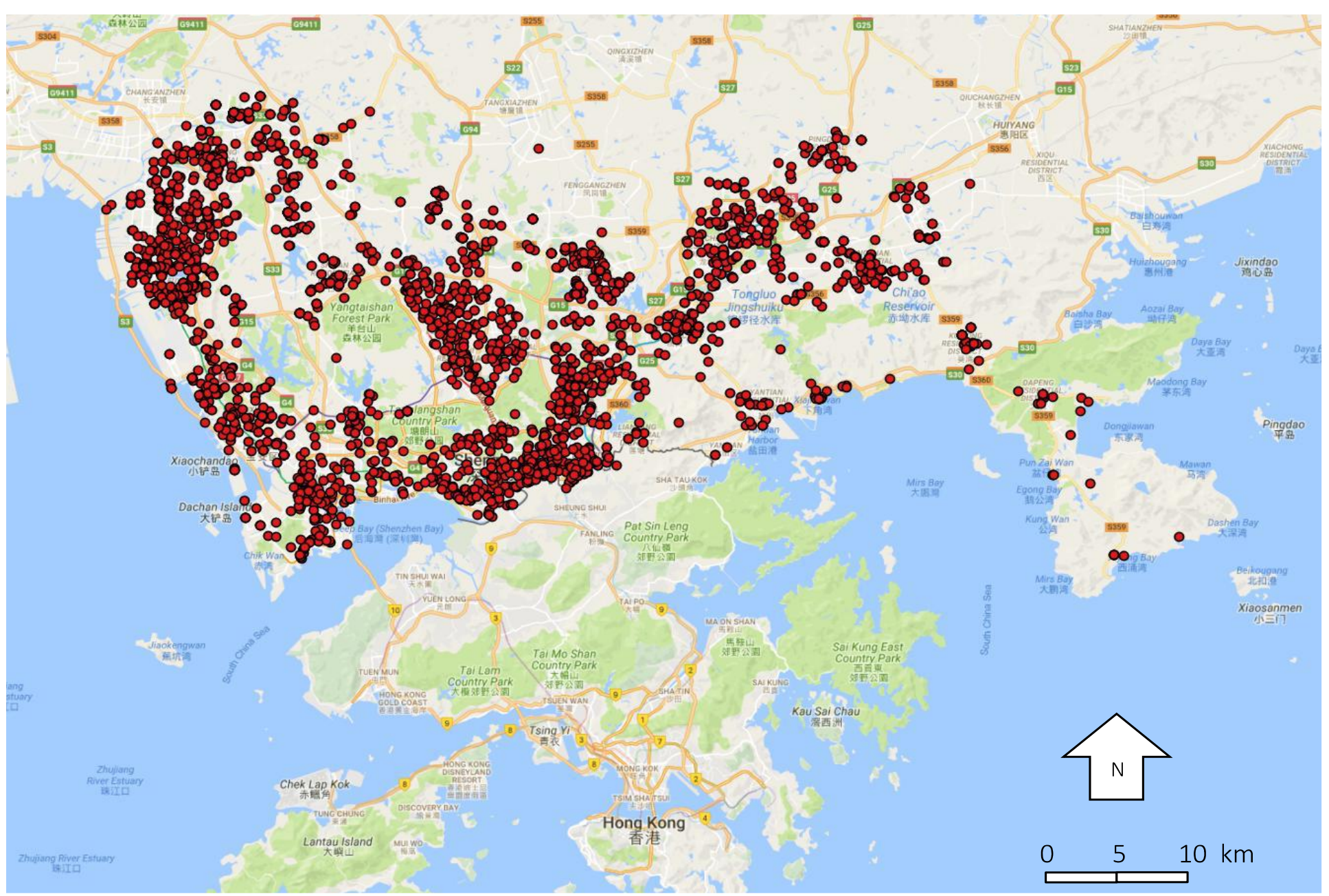


Figure 3. Kernel density estimate map of incidents which met criteria for Step 1 or 2 of the Field Triage Decision Scheme, and hospitals. Blue stars indicate teaching hospitals, yellow diamonds regional hospitals, pink squares community hospitals, and white circles private hospitals.

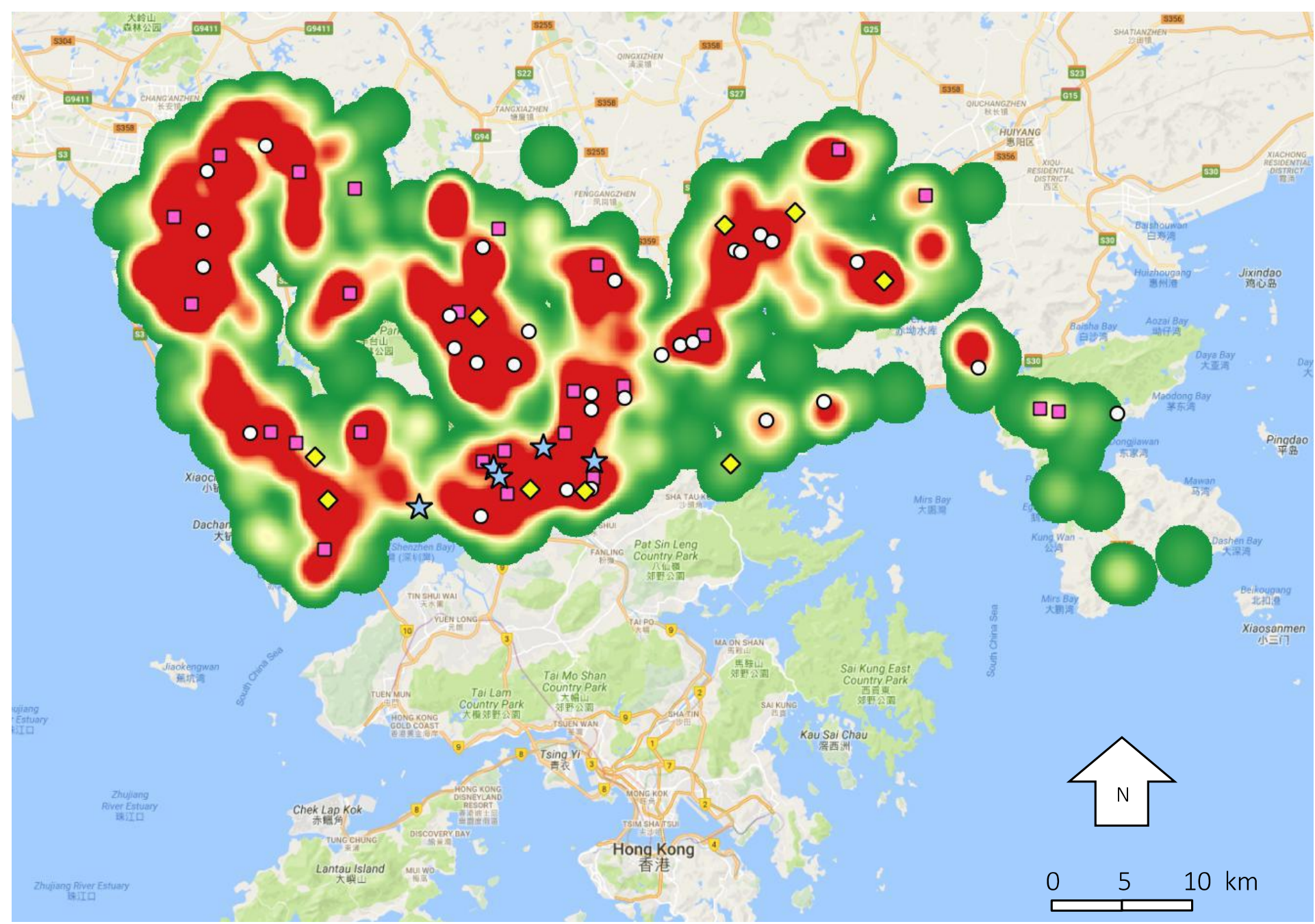

